\title{
Critical behavior of the energy and pressure correlation functions in SU(2) gauge theory
}

\author{
C. Pica* ${ }^{*}$ \\ Brookhaven National Laboratory \\ Physics Department, Brookhaven National Laboratory, Upton, NY 11973, USA \\ E-mail: pica@quark • phy . bn l.gov

\section{K. Hübner} \\ Brookhaven National Laboratory \\ Physics Department, Brookhaven National Laboratory, Upton, NY 11973, USA \\ E-mail: huebner@quark • phy . bnl.gov

\section{F. Karsch} \\ Brookhaven National Laboratory \\ Physics Department, Brookhaven National Laboratory, Upton, NY 11973, USA \\ Fakultät für Physik, Universität Bielefeld, D-33615 Bielefeld, Germany \\ E-mail: karsch@quark.phy.bnl.gov
}

\begin{abstract}
The critical behavior of zero-momentum correlation functions of the energy-momentum tensor components near the second order finite temperature transition of the (3+1)-dimensional SU(2) gauge theory is studied. While the correlation function of the trace of the energy-momentum tensor is shown to diverge uniformly like the specific heat as the critical temperature is approached, the pressure-pressure correlation function remains finite. Implications relevant for the calculation of transport coefficients, in particular the bulk viscosity, in the vicinity of a second order phase transition are discussed.
\end{abstract}

The XXVI International Symposium on Lattice Field Theory

July 14-192008

Williamsburg, Virginia, USA

\footnotetext{
* Speaker.

$\dagger$ address after Oct. 01: School of Physics, University of Edinburgh, Edinburgh EH9 3JZ, UK
} 


\section{Introduction}

\subsection{Motivation}

The experimental evidence for rapid thermalization of the dense matter created in heavy ion collisions at RHIC has led to the interpretation of the quark gluon plasma above but close to the transition temperature as a strongly interacting medium that has properties of an almost perfect liquid. These experimental findings also renewed the interest in determining transport properties of gauge theories through the calculation of correlation functions of the energy-momentum tensor on the lattice. Recently it has been argued that close to the transition from low temperature hadronic matter to the plasma phase of QCD bulk viscosity might play a much more important role than shear viscosity. The singular behavior of bulk viscosity in the vicinity of a critical point has long been known in statistical physics. In particular, at the critical point of the liquid gas transition it has been argued that the divergence of $\zeta$ is strong and, in fact, almost quadratic in the inverse reduced temperature $t$. The singular behavior, $\zeta \sim t^{-z v+\alpha}$, with $\alpha, v$ denoting static critical exponents of the 3-d Ising model and $z$ being a dynamical exponent characterizing the equilibration of density fluctuations, goes along with a strong divergence of the relaxation time for density fluctuations, $\tau_{R}$. Their ratio, $\zeta / \tau_{R} \sim t^{\alpha}$, however, is proportional to the inverse of the specific heat and thus vanishes slowly at the critical point. The SU(2) gauge theory with its second order deconfinement phase transition seems to be an ideal model to explore critical behavior of dynamical properties, e.g. transport coefficients. It also may give insight into transport properties in the vicinity of the chiral critical point in QCD that may exist at non-zero baryon number density and also belongs to the Ising universality class. Many of the results presented in this proceeding, have been discussed in more detail in [1].

\subsection{Energy-Momentum tensor and bulk viscosity}

We indicate with $\Theta^{\mu v}$ the energy-momentum tensor. The energy density is $\varepsilon=\Theta^{00}$, and the pressure is given by $3 p=-\Theta^{i i}$. Given a zero-momentum connected correlation function at finite temperature $T: G_{X Y}(\tau, T)=\int d^{3} x\langle X(\vec{x}, \tau) Y(\overrightarrow{0}, 0)\rangle_{T}$, its spectral function $\rho_{X Y}$ is given by: $G_{X Y}(\tau, T)=\int d \omega \rho_{X Y}(\omega, T) \cosh [\omega(\tau-1 / 2 T)] \operatorname{cosech}(\omega / 2 T)$.

The bulk viscosity can be extracted from the low frequency behavior of the spectral function of the pressure-pressure correlator:

$$
\zeta(T)=\pi \lim _{\omega \rightarrow 0} \frac{\rho_{p p}(\omega, T)}{\omega} .
$$

\section{SU(2) thermodynamics}

\subsection{Differential formalism}

In order to analyze correlation functions of $\Theta^{\mu \mu}$ we need to work with a formulation of Euclidean thermodynamics that allows to introduce local operators for energy density and pressure. This is naturally achieved within the differential formalism for bulk thermodynamics [2]. We introduce the Euclidean action on an anisotropic lattice as,

$$
S=\frac{2 N}{g_{\sigma}^{2}} \sum_{x, i>j=1,2,3}\left(1-\frac{1}{N} \operatorname{ReTrP}_{\mathrm{ij}}(\mathrm{x})\right)+\frac{2 N}{g_{\tau}^{2}} \sum_{x, j=1,2,3}\left(1-\frac{1}{N} \operatorname{Re}_{\operatorname{Tr}} \mathrm{P}_{4 \mathrm{j}}(\mathrm{x})\right) .
$$


Thermodynamic quantities can be obtained taking derivatives of the free energy, e.g.:

$$
\varepsilon=-\frac{1}{V} \frac{\partial \ln Z}{\partial(1 / T)}-\varepsilon_{0}, \quad p=T \frac{\partial \ln Z}{\partial V}-p_{0},
$$

where we have subtracted the vacuum contribution of $\mathrm{T}=0$. On an isotropic lattice these are:

$$
\begin{aligned}
& \frac{\varepsilon}{T^{4}}=N N_{\tau}^{4}\left\{3\left[2 g^{-2}-\left(c_{\sigma}-c_{\tau}\right)\right]\left(P_{\sigma}-P_{\tau}\right)+3\left[c_{\sigma}+c_{\tau}\right]\left(2 P_{0}-P_{\sigma}-P_{\tau}\right)\right\} \\
& \frac{p}{T^{4}}=N N_{\tau}^{4}\left\{\left[2 g^{-2}-\left(c_{\sigma}-c_{\tau}\right)\right]\left(P_{\sigma}-P_{\tau}\right)-3\left[c_{\sigma}+c_{\tau}\right]\left(2 P_{0}-P_{\sigma}-P_{\tau}\right)\right\}
\end{aligned}
$$

and $\Theta^{\mu \mu} \equiv \varepsilon-3 p$. In the above equations we have:

$$
\begin{gathered}
g^{-2}=g_{\sigma}^{-2}=g_{\tau}^{-2} ;\left.B\left(g^{-2}\right) \equiv \frac{d g^{-2}}{d \ln a}\right|_{\xi=1}=-2\left(c_{\sigma}+c_{\tau}\right) ; c_{\sigma, \tau}=\left.\frac{\partial g_{\sigma, \tau}^{-2}}{\partial \xi}\right|_{\xi=1} \\
P_{\sigma}=\frac{1}{3 N_{\sigma}^{3} N_{\tau}} \sum_{x, i>j=1,2,3}\left(1-\frac{1}{N} \operatorname{ReTr}_{\mathrm{ij}}(\mathrm{x})\right) ; P_{\tau}=\frac{1}{3 N_{\sigma}^{3} N_{\tau}} \sum_{x, j=1,2,3}\left(1-\frac{1}{N} \operatorname{ReTrP}_{4 \mathrm{j}}(\mathrm{x})\right)
\end{gathered}
$$

\subsection{Critical energy density and pressure}

As check of our setup we perform a finite-size scaling analysis of $\varepsilon$ and $p$ at the critical point. From the scaling ansatz for the singular part of the free energy density $\left(t \equiv\left(T-T_{c}\right) / T_{c}\right)$ : $f_{s}\left(t, L^{-1}\right)=b^{-d} f_{s}\left(t b^{1 / v}, L^{-1} b\right)$, it follows for the energy density and pressure $(\alpha=2-d v)$ :

$$
\begin{aligned}
& \left(\frac{p\left(T_{c}\right)}{T_{c}^{4}}\right)_{N_{\tau}, N_{\sigma}}=\left(\frac{p\left(T_{c}\right)}{T_{c}^{4}}\right)_{N_{\tau}, \infty}+a_{p} N_{\sigma}^{-3}, \\
& \left(\frac{\varepsilon\left(T_{c}\right)}{T_{c}^{4}}\right)_{N_{\tau}, N_{\sigma}}=\left(\frac{\varepsilon\left(T_{c}\right)}{T_{c}^{4}}\right)_{N_{\tau}, \infty}+a_{\varepsilon} N_{\sigma}^{-(1-\alpha) / v} .
\end{aligned}
$$

The critical exponents $\alpha=0.110(1)$ and $v=0.6301(4)$ are known from the $3 \mathrm{~d}$ Ising model. From the critical behavior of $\varepsilon$ and $p$ it follows that for generic combinations of the two, like $\varepsilon-3 p$ and $\varepsilon+p$, will have the same volume scaling as the energy density. Note also that the value of $P_{0}$, entering in the above expressions, is inessential in the analysis of finite-size scaling at fixed $N_{\tau}$, since it can be considered as a constant. We used lattices with $N_{\tau}=4,6,8$ and $N_{\sigma} / N_{\tau}$ up to 24 $\left(96^{3} \times N_{\tau}\right)$. The infinite volume critical couplings are already known: $\beta_{c}=2.29895(10)$ for $N_{\tau}=4$ [3], $\beta_{c}=2.4265(30)$ for $N_{\tau}=6$ [4], and $\beta_{c}=2.5115(40)$ for $N_{\tau}=8$ [5].

To check the universality class, using the $N_{\tau}=4$ lattices, where more volumes are available, we determined the critical exponent $(1-\alpha) / v$ by a fit to the expected functional form of the energy density. The fit yields $(1-\alpha) / v=1.41(6)$ in agreement with $(1-\alpha) / v=1.412(1)$ for Ising $3 \mathrm{~d}$. Using the known values for the critical exponents, we extracted the infinite volume critical $\varepsilon$ and $p$ Fig. 1. The results are summarized in Table 1. The fit was done fixing the known value of the critical exponents and using only lattices with $N_{\sigma} / N_{\tau}>4$. The result for $\varepsilon\left(T_{c}\right) / T_{c}^{4}$ is compatible with the value $\varepsilon\left(T_{c}\right) / T_{c}^{4}=0.256(23)$ from [2]. The result for the critical pressure is new.

\section{Correlation functions of $\Theta^{\mu \mu}$}

The correlation function for the trace of the energy-momentum tensor can be decomposed as:

$$
G_{\Theta \Theta}(\tau, T)=G_{\varepsilon \varepsilon}(\tau, T)-6 G_{\varepsilon p}(\tau, T)+9 G_{p p}(\tau, T) .
$$




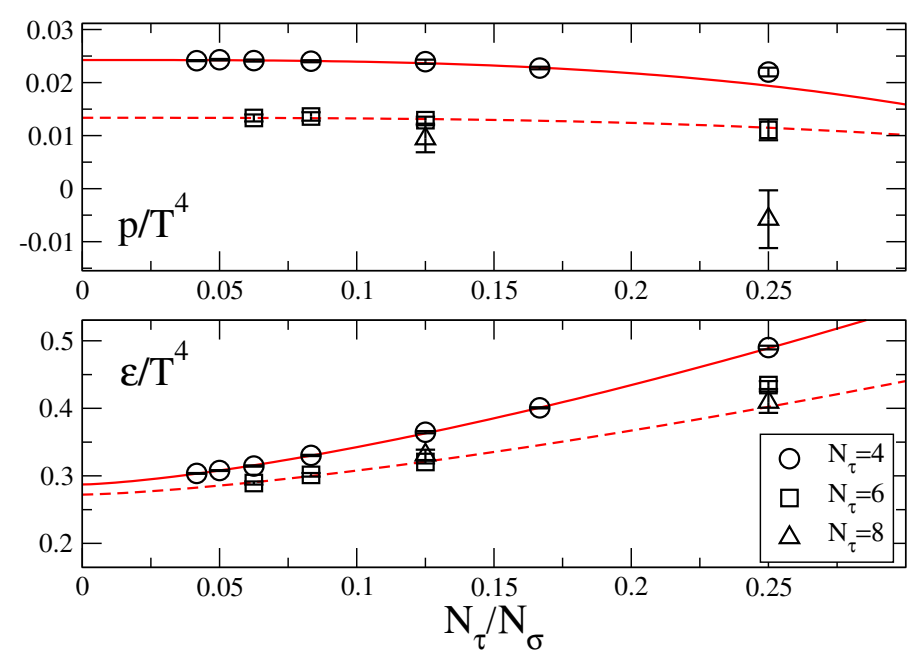

Figure 1: Critical behavior of the energy density and pressure. The difference in their behavior is evident from the size of the relative deviations at finite volume. Notice also the small absolute value of the pressure at the critical point.

\begin{tabular}{c|l|l}
$N_{\tau}$ & $\varepsilon\left(T_{c}\right) / T_{c}^{4}$ & $P\left(T_{c}\right) / T_{c}^{4}$ \\
\hline 4 & $0.28724(53)$ & $0.02423(30)$ \\
6 & $0.2722(31)$ & $0.0135(8)$ \\
8 & - & $0.0107(15)$ \\
\hline
\end{tabular}

Table 1: Energy density and pressure at infinite volume as extrapolated from our data, for different $N_{\tau}$. Large volumes and statistics allow for an unprecedented accuracy in the determination of $\varepsilon$ and for the first time of the pressure at the critical point.

The correlation functions involving the energy density operator $G_{\varepsilon Y}(\tau, T)$ are independent of $\tau$ in the continuum limit. This is easily seen since $\langle H(\tau) Y(0)\rangle_{T}=\frac{1}{Z} \operatorname{Tr}\left[e^{-H / T} H(\tau) Y(0)\right]$ is independent on time. It also follows that: $G_{\varepsilon Y}(\tau, T)=-\frac{\partial}{\partial(1 / T)}\langle Y\rangle_{T}$. The whole $\tau$ dependence of $G_{\Theta \Theta}$ is thus expected to arise from the pressure-pressure correlations. On the other hand, the dominant temperature dependence comes from the energy-energy correlator, which is proportional to the specific heat: $\frac{G_{\varepsilon \varepsilon}(\tau, T)}{T^{5}} \sim \frac{c_{V}}{T^{3}}$. At non-zero lattice spacing the direct relation between correlation functions involving the energy operator and temperature derivatives of any observable is violated by cut-off effects. Nonetheless we expect that these are small in the vicinity of a second order phase transition. One thus may expect that at least the singular behavior of correlation functions that involve correlations with the energy operator will be independent of Euclidean time. Close to the deconfinement transition we therefore expect that $G_{\Theta \Theta}$ will show, for every $\tau$, a critical behavior that coincides with that of the specific heat in a 3-dimensional Ising model:

$$
\frac{G_{\Theta \Theta}(\tau, T)}{T^{5}} \sim \frac{c_{V}}{T^{3}} \sim A_{ \pm}\left|\frac{T-T_{c}}{T_{c}}\right|^{-\alpha}\left(1+B_{ \pm}\left|\frac{T-T_{c}}{T_{c}}\right|^{\omega}+\ldots\right) \quad \text { for } \quad T \rightarrow T_{c}^{ \pm}
$$

with known universal exponents: $\alpha=0.110(1), v=0.6301(4)$. Like $\alpha, v$ also the ratio of amplitudes $A_{+} / A_{-}=0.54(1)$ and the correction to scaling exponent $\omega / v=0.84(4)$ are universal. 

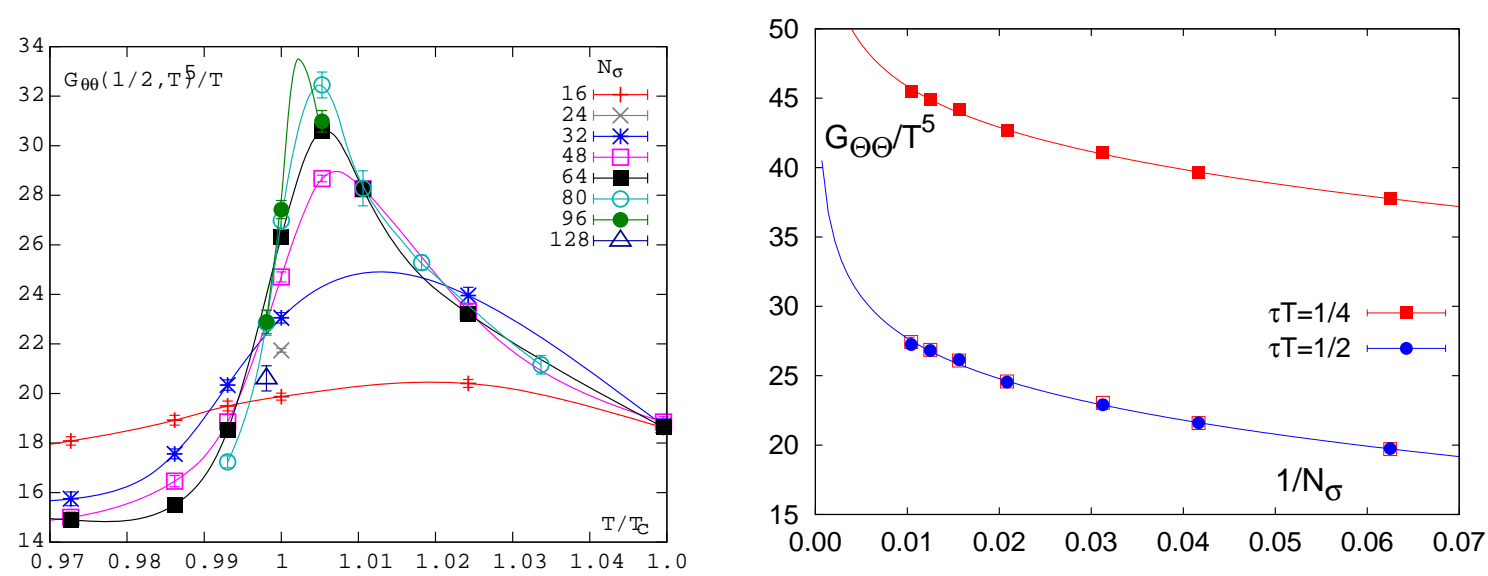

Figure 2: Left: $G_{\Theta \Theta}$ for different volumes at $\tau T=1 / 2$. Right: combined fit using Eq.(3.3) at $T=T_{c}$.

We have calculated the correlation function $G_{\Theta \Theta}(\tau, T)$ close to the deconfinement transition point of the $\mathrm{SU}(2)$ gauge theory. In our simulation we use lattices of size $N_{\sigma}^{3} N_{\tau}$ with $N_{\tau}=4$. This gives us information on the correlation function at two non-zero values of Euclidean time, i.e. at $\tau T=1 / 4$ and at the midpoint $\tau T=1 / 2$. Most of our simulations have been performed at temperatures close to the phase transition where the correlation length becomes large. This required calculations on large spatial lattices in order to eliminate finite volume effects. We used spatial lattice sizes with aspect ratios $N_{\sigma} / N_{\tau}$ varying from $8\left(32^{3} \times 4\right.$ lattices) up to values as large as 32 $\left(128^{3} \times 4\right.$ lattices). A large number of configurations are required to reach the statistical accuracy $-\mathscr{O}(5 \%)$ at $\tau T=1 / 2-$ needed for scaling test near the critical point. We have generated about $1 \cdot 10^{6}$ configurations on our smaller lattices and up to $4 \cdot 10^{5}$ on the large lattices. The algorithm used to generate the configurations uses a standard mixture of heat-bath and over-relation updates in a typical ratio of 1:4-1:6 to keep correlations between consecutive configurations small. Autocorrelation times in the transition region range from $\mathscr{O}(1)$ on the small lattices to about 40 on the largest lattices. Numerical simulations have been performed on the BlueGene/L, BlueGene/P at the New York Center for Computational Science (NYCCS), using a code developed specifically for this work. A sample of our dataset for $G_{\Theta \Theta}$ at $\tau T=1 / 2$ is shown in Fig. 2 (left).

At $T_{c}$ the singular part behavior of $G_{\Theta \Theta}$ is expected to be:

$$
G_{\Theta \Theta}\left(\tau, T_{c}\right) / T_{c}^{5}=A_{\sigma} N_{\sigma}^{\alpha / v}\left(1+B_{\sigma} N_{\sigma}^{-\omega / v}\right)+C_{\sigma},
$$

where $A_{\sigma}, B_{\sigma}, C_{\sigma}$ might a priori depend on Euclidean time. This functional form gives excellent fits for both datasets at distance $\tau T=1 / 4$ and $\tau T=1 / 2$, and the fit indicates that at $T_{c}$ the singular contributions to $G_{\Theta \Theta}(\tau, T)$ are independent on the Euclidean time separation $\tau$, see Fig. 2 (right).

In the vicinity of $T_{c}$ we expect the data to be well described by the scaling ansatz,

$$
G_{\Theta \Theta}(\hat{\tau}, T) / T^{5}=A_{ \pm} t^{-\alpha}\left(1+B_{ \pm} t^{\omega}\right)+C+D t
$$

where $A_{+}, B_{ \pm}, C, D$ are free parameters, which again all may depend on Euclidean time. This provides very good fits in the interval $T / T_{c} \in[0.94,1.05]$. Again we find that within errors the fit parameters $A_{+}$and $B_{ \pm}$are independent of $\tau$; a combined fit of $G_{\Theta \Theta}(1 / 4, T) / T^{5}$ and $G_{\Theta \Theta}(1 / 2, T) / T^{5}$ 


\begin{tabular}{|c|c|c|c|c|c|c|c|c|}
\hline$\tau T$ & $A_{+}$ & $B_{+}$ & $B_{-}$ & $C$ & $D$ & $A_{\sigma}$ & $B_{\sigma}$ & $C_{\sigma}$ \\
\hline \multicolumn{9}{|c|}{ free fit } \\
\hline $1 / 4$ & $\begin{array}{l}16.3(2.1) \\
\end{array}$ & $-0.59(19)$ & $-1.9(1.4)$ & $21.5(5.7)$ & $-150(53)$ & $9.2(1.2)$ & $-2.4(1.0)$ & $26.3(2.7)$ \\
\hline $1 / 2$ & $16.4(2.2)$ & $-0.76(18)$ & $-2.2(1.5)$ & $3.7(3.4)$ & $-95(32)$ & $9.1(1.2)$ & $-2.4(0.9)$ & $8.4(2.9)$ \\
\hline \multicolumn{9}{|c|}{ combined fit } \\
\hline $\begin{array}{l}1 / 4 \\
1 / 2\end{array}$ & $16.5(1.3)$ & $-0.74(10)$ & $-2.12(86)$ & $\begin{array}{c}21.8(2.1) \\
3.8(1.9)\end{array}$ & $\begin{array}{c}-143(20) \\
-87(18)\end{array}$ & $9.15(73)$ & $-2.39(59)$ & $\begin{array}{c}26.4(1.9) \\
8.3(1.7)\end{array}$ \\
\hline
\end{tabular}

Table 2: Fit parameters for fits to $G_{\Theta \Theta}(\tau, T) / T^{5}$ using Eq.(3.3) and (3.4). The last row gives parameters of combined fits to the data for $\tau T=1 / 4$ and $1 / 2$.
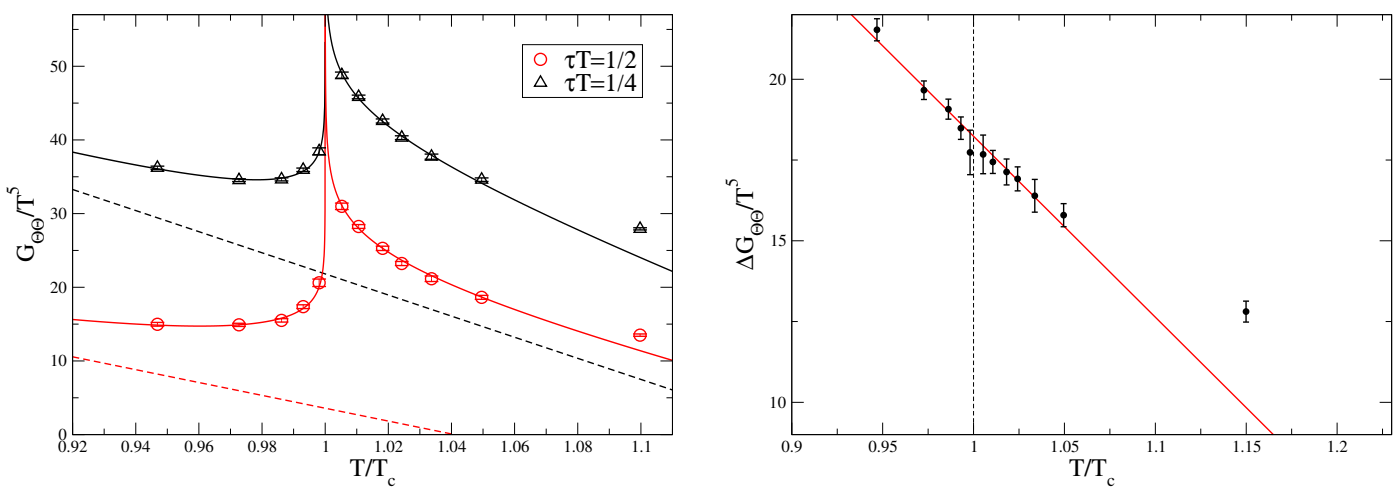

Figure 3: Infinite volume $G_{\Theta \Theta}$ near $T_{c}$. Left: combined fit to the ansatz Eq.(3.4); right: the difference $G_{\Theta \Theta}(1 / 4, T)-G_{\Theta \Theta}(1 / 2, T)$.

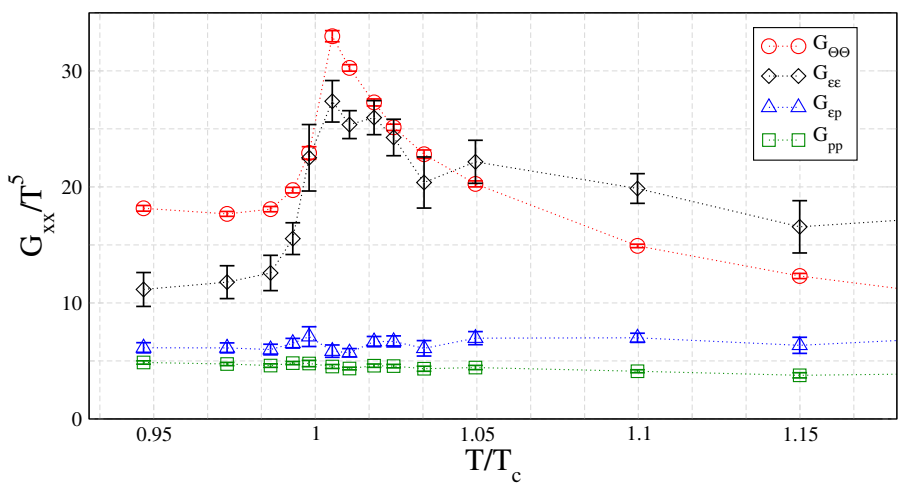

Figure 4: Decomposition of $G_{\Theta \Theta}$ into different contributions as in Eq.(3.1).

with common amplitudes $A_{+}$and $B_{ \pm}$also gives a $\chi^{2} / d o f=1.1$, see Fig. 3 (left). A summary of the fit results can be found in Table 2. In order to eliminate the leading singular behavior from $G_{\Theta \Theta}$ it thus suffices to consider $\Delta G_{\Theta \Theta}(\tau, T) \equiv G_{\Theta \Theta}(\tau, T)-G_{\Theta \Theta}(1 / 2 T, T)$. Fig. 3 (right) explicitly shows that the singular term in $G_{\Theta \Theta}$ gives a contribution to its spectral function which is proportional to a delta function at zero frequency.

We finally show in Fig. 4 the temperature dependence of the different correlation functions, $G_{X X}(\tau, T)$, contributing to $G_{\Theta \Theta}$. As can be seen the entire strong temperature dependence of $G_{\Theta \Theta}$ 
in the vicinity of $T_{c}$ indeed arises from $G_{\varepsilon \varepsilon}$. All other correlators show only a weak temperature dependence in the vicinity of $T_{c}$. In particular, we find that the pressure-pressure correlation function stays finite at $T_{c}$ and varies little in its vicinity and does not present any singular behavior. From this observation we cannot conclude however that the bulk viscosity itself is not diverging. In fact consider a simple ansatz for the form of the spectral function: $\rho_{p p}(\omega, T) \equiv f\left(\omega, \zeta, \omega_{0}\right)+\rho_{>}(\omega, T)$, where the low-frequency part is modeled by a Breit-Wigner: $f\left(\omega, \zeta, \omega_{0}\right)=\frac{1}{\pi} \zeta \frac{\omega \omega_{0}^{2}}{\omega^{2}+\omega_{0}^{2}}$, with $\zeta$ and $\omega_{0}$ both dependent only on $T$. If approaching $T_{c}, \omega_{0} \rightarrow 0$ we have:

$$
G_{p p}(\tau, T) \propto T \zeta(T) \omega_{0}(T)+\mathscr{O}\left(\omega_{0}^{2}\right)+\text { high frequency part } .
$$

We have therefore that the product $\zeta(T) \omega_{0}(T)$ remains finite at the critical temperature, while the bulk viscosity itself may or may not diverge. The parameter $\omega_{0}$ represent the characteristic frequency range over which $\rho / \omega$ remains constant and equal to $\zeta$. Assuming that $\omega_{0}$ is related to the inverse relaxation time we have: $\omega_{0}(t) \propto t^{z v}$, where $z$ is a dynamical critical exponent $z \sim 2-3$. This in turn implies that it is still possible that the bulk viscosity is rather strongly divergent at $T_{c}$ while no visible singularity is visible in the pressure-pressure correlator.

\section{Conclusions}

As a preliminary step, we have studied the thermodynamic properties of the SU(2) LGT at the deconfinement transition. Specifically the critical behavior of the pressure, energy density was analyzed and found in excellent agreement with the expected 3-d Ising critical behavior. An estimate for the critical $\varepsilon$ and $p$ in the continuum limit was given; We have shown that the correlation function of the trace of the energy-momentum tensor $G_{\Theta \Theta}$ diverges at the critical temperature. Using the finite size scaling at the critical temperature and the temperature scaling in the critical region, the singular structure of $G_{\Theta \Theta}$ was found to be consistent, with high accuracy, with that of the specific heat $c_{V}$. We have shown that $G_{\Theta \Theta}$ becomes independent of Euclidean time at the critical point, which indicates that its spectral representation has a $\delta$-function singularity at zero frequency. The correlators $G_{\varepsilon \varepsilon}, G_{\varepsilon p}$ and $G_{p p}$ were also analyzed. The singular behavior of $G_{\Theta \Theta}$ can be traced back in the corresponding singular behavior of $G_{\varepsilon \varepsilon}$, while the other two correlation functions remain finite at the critical point. It turned out however that to extract the behavior of the bulk viscosity a much more subtle analysis must be done: namely one must be able to control the width of the peak of the spectral function at low frequencies but unfortunately at present no such analysis exists.

\section{References}

[1] K. Huebner, F. Karsch and C. Pica, arXiv:0808.1127 [hep-lat].

[2] J. Engels, F. Karsch and K. Redlich, Nucl. Phys. B 435, 295 (1995).

[3] J. Engels and T. Scheideler, Nucl. Phys. B 539, 557 (1999).

[4] J. Engels, J. Fingberg and D. E. Miller, Nucl. Phys. B 387 (1992) 501.

[5] J. Fingberg, U. Heller and F. Karsch, Nucl. Phys. B 392, 493 (1993). 